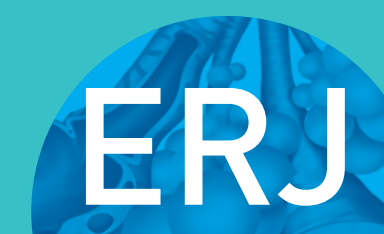

open research
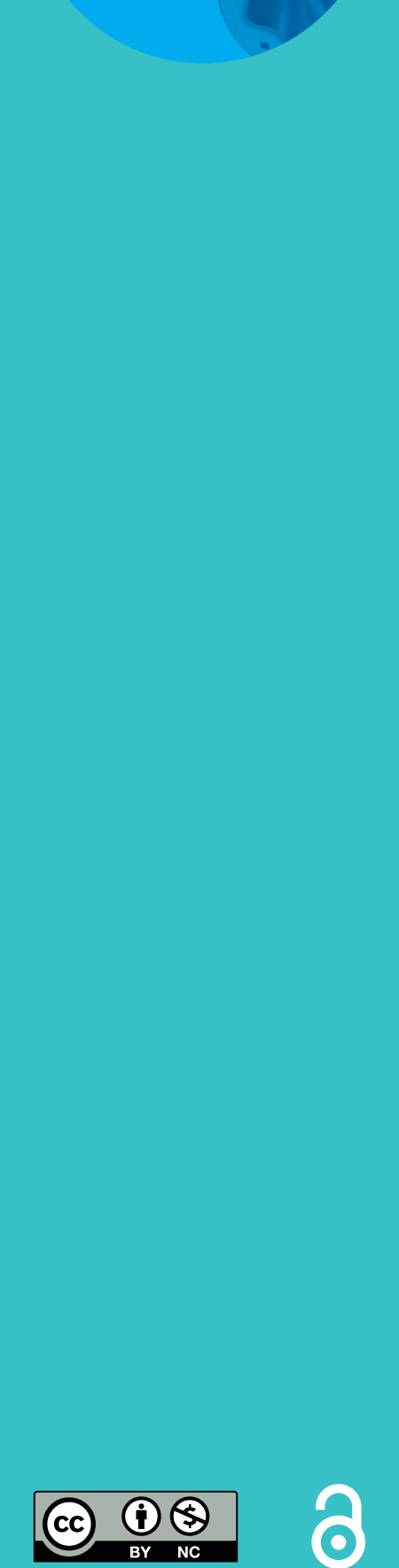

\section{Psychological profile of individuals presenting with chronic cough}

\author{
Katrin Hulme ${ }^{1,2,3}$, Vincent Deary ${ }^{4}$, Sian Dogan $^{1}$ and Sean M. Parker ${ }^{1}$
}

Affiliations: ${ }^{1}$ Respiratory Medicine, North Tyneside Hospital, Northumbria Healthcare NHS Foundation Trust, North Shields, UK. ${ }^{2}$ Health Psychology, Guy's Hospital, King's College London, London, UK. ${ }^{3}$ Health Psychology, Staffordshire University, Stoke-on-Trent, UK. ${ }^{4}$ Psychology Dept, Northumbria University, Newcastle, UK.

Correspondence: Sean Parker, Consultant Respiratory Physician, Department of Respiratory Medicine, Northumbria Healthcare NHSFT, North Tyneside General Hospital, Rake Lane, North Shields NE29 8NH, UK. E-mail: sean.parkerlanhct.nhs.uk

ABSTRACT Chronic refractory cough (CRC) is a common problem in respiratory clinics. Adverse effects on quality of life are documented in the literature, but relatively little is known about the underlying psychological factors in this patient population. We aimed to investigate the association of psychological factors with chronic cough, comparing CRC to explained cough and non-cough groups.

67 patients attending a specialist cough clinic (CRC, $n=25$; explained cough, $n=42$ ) and 22 non-cough individuals participated. All participants completed the Hospital Anxiety \& Depression Scale, Big Five Inventory (Personality), Chalder Fatigue Scale and Patient Health Questionnaire-15. Cough patients also completed the Illness Perception Questionnaire-Revised. Appropriate statistical analyses were used to compare participant groups.

Chronic refractory coughers displayed significantly higher levels of anxiety, depression, fatigue and somatic physical symptoms than non-cough participants. Compared to explained coughers, there were higher depression and fatigue scores and significantly more negative illness representations (specifically, strong beliefs regarding negative consequences, lower illness coherence and higher emotional representations). "Explained" coughers reported significantly increased fatigue and somatic symptoms in comparison to non-coughers.

The prevalence of fatigue, low mood, negative illness beliefs and increased physical symptom reporting should be considered in consultations and in developing novel interventions for CRC patients.

@ERSpublications

Refractory cough is associated with psychological factors, such as low mood, illness beliefs and fatigue http://ow.ly/hbKt30923pb

Cite this article as: Hulme $\mathrm{K}$, Deary V, Dogan S, et al. Psychological profile of individuals presenting with chronic cough. ERJ Open Res 2017; 3: 00099-2016 [https://doi.org/10.1183/ 23120541.00099-2016].

Received: Sept 162016 | Accepted after revision: Jan 062017

Support statement: This work was funded by Northumbria Healthcare NHSFT. Funding information for this article has been deposited with the Crossref Funder Registry.

Conflict of interest: None declared.

Copyright $\odot$ ERS 2017. This article is open access and distributed under the terms of the Creative Commons Attribution Non-Commercial Licence 4.0. 


\section{Introduction}

Most patients who present with a persistent cough find the symptom resolves either naturally with time or after a series of empirical treatment trials [1]. However, a significant number of patients (12-46\%) have a treatment-resistant, chronic refractory cough (CRC) with no evident cause [2].

Regardless of cause, a cough can disrupt various aspects of everyday life, such as sleep, social activities and communication [3, 4]. A questionnaire study concluded that chronic coughers have increased levels of emotional distress compared to a healthy population, although there was no non-cough comparison group [5]. There was no significant difference between explained and unexplained apart from state anxiety. A similar study confirmed a higher than expected prevalence of depression in chronic cough, but did not distinguish between refractory and explained coughers [6]. Improvements in cough symptoms after treatment correlated with depression scores, possibly implying a causal link between cough impact on quality of life and psychological wellbeing [10].

Research has shown that the cough reflex is more than a simple, protective reflex; it can be modulated by attentional focus [7] and harmful information processing [8], as well as be voluntarily supressed or modified [9]. Furthermore, functional magnetic resonance imaging (MRI) studies have mapped "urge to cough" and cough suppression onto higher-order brain regions [10]. This evidence suggests a role for higher-order, psychological processes in cough, highlighting a possible alternative avenue for cough treatment.

However, although current evidence for non-pharmacological treatment suggests it can be effective [11, 12], the mechanisms of action are unclear. Increasing our understanding of the factors associated with the condition is not only important for its own sake, but also to inform high-quality systematic intervention development in line with the Medical Research Council guidelines for complex intervention development [13].

Psychosocial factors implicated in the onset or maintenance of other persistent physical symptoms have the potential to contribute to our understanding of CRC. Personality variables, specifically neuroticism, have been associated with a number of physical and mental health markers, both phenotypically and genetically [14], including other so-called medically unexplained symptoms (MUSs) [15]. Although neuroticism is of particular interest given the psychomorbidity findings reported previously [5, 6], all personality dimensions are worth investigating.

Somatic symptoms often cluster and overlap. For example, irritable bowel syndrome (IBS) patients often report lower back pain, muscle pain, difficulty concentrating, disturbed sleep and joint problems, symptoms more commonly associated with chronic fatigue syndrome (CFS). Conversely, CFS patients often suffer from the gastrointestinal symptoms associated with IBS [16]. The association of IBS symptoms and chronic cough has also been noted [17], so investigating the presence of other somatic symptoms is warranted.

Fatigue has been measured in the cough literature but only as a subscale of a quality-of-life dimension. Terms such as "exhaustion" and "sleep disturbance" appear in the cough literature [3, 4]. However, we are not aware of research using a specific scale to quantify fatigue. Given the potentially detrimental impact of fatigue on everyday functioning and coping [18], if it is prevalent then it should be considered during intervention development.

Illness perceptions are an individual's cognitive representation of their illness: its causes, associated symptoms, consequences, timeline, controllability, emotional impact and conceptual coherence [19]. These beliefs guide how the person understands and manages their illness. For example, negative consequence beliefs (adverse impact of the condition), reduced illness coherence (an individual's understanding of the illness) and heightened emotional representations (an individual's emotional reactions to the health condition) have been shown to predict reduced quality of life in IBS $[20,21]$. These factors may also be salient for CRC.

The aim of this exploratory study is to investigate psychological factors associated with chronic cough. Specifically, we hypothesise that compared to explained coughers and non-cough participants, CRC patients will have higher levels of negative affectivity, fatigue and negative illness perceptions.

\section{Methods}

Design

A cross-sectional questionnaire survey design was employed. Differential statistical analyses established whether associations existed between the constructs and chronic cough, and investigated any differences among the three groups.

\section{Participants}

Participants were consecutively recruited from a secondary-care specialist cough clinic at North Tyneside Hospital between September 2014 and February 2015. Participating patients were also asked to take a questionnaire bundle for a "non-cough" friend to complete; this was our healthy, non-cough comparison 
group. Inclusion criteria for clinic patients were: $\geqslant 18$ years old and chronic cough $(>8$ weeks, either "refractory" or "explained"). For the non-cough participants, the inclusion criteria were: $\geqslant 18$ years old, no chronic cough and not currently attending hospital appointments. Exclusion criteria were: unable to give informed consent, serious coexistent psychiatric disorder and coexistent illness. Explained coughers were defined as those who had a medical diagnosis to explain why they were coughing (for instance asthma, chronic obstructive pulmonary disease (COPD) or interstitial lung disease) on the basis of clinical assessment and response, in some cases, to treatment. Refractory coughers were those patients with no objective pulmonary pathology who had not responded to trials of treatment. Patients were assigned to these groups retrospectively following a review of the medical notes by the investigators.

\section{Questionnaires}

The following five validated questionnaires were utilised to measure their respective constructs: the Big Five Inventory (BFI), the Chalder Fatigue Scale, the Patient Health Questionnaire-15 (PHQ-15), the Hospital Anxiety and Depression Scale (HADS) and the Illness Perception Questionnaire-Revised (IPQ-R).

\section{Big Five Inventory}

The BFI is a 44-item inventory measuring the "Big Five" personality dimensions: openness, conscientiousness, extraversion, agreeableness and neuroticism. Statements are rated using a 5-point Likert scale: 1, "Disagree strongly" to 5, "Agree strongly". This measure has good internal reliability (mean subscale Cronbach's $\alpha=0.83$ ) [22].

\section{Chalder Fatigue Scale}

11 items are scored on a four-point scale from "less than usual (0)" to "much more than usual (3)". The higher the summed score, the more fatigued the individual. This measure has been validated in a community sample (mean=14.2) and has high internal reliability; Cronbach's $\alpha=0.92$ [23].

\section{Patient Health Questionnaire-15}

15 items address somatic, physical symptoms in the past 4 weeks. Each response is rated on a 3-point Likert scale from "not bothered at all (0)" to "bothered a lot (2)". Scores are summed and higher scores represent increased somatic symptom severity. Cronbach's $\alpha=0.80$ and cut-off scores are 5 (mild), 10 (moderate) and 15 (severe) [24].

\section{Hospital Anxiety and Depression Scale}

HADS is a 14-item scale (seven addressing anxiety (A) and seven for depression (D)), scored from 0 to 3 [25]. A score of 8 or more has been identified as the borderline cut-off point and 11+ indicates probable disorder. Internal consistency for this measure is adequate to excellent with Cronbach's $\alpha$ ranging between 0.68 and 0.93 for the HADS-A subscale and 0.67 to 0.90 for the HADS-D subscale [26].

\section{Illness Perception Questionnaire-Revised}

This questionnaire assesses peoples' illness beliefs on nine dimensions: identity, cause, consequences, timeline cyclical and chronic, personal and treatment control, emotional representations and illness coherence [27]. Identity is assessed by "yes" (1) or "no" (0) responses. Other dimensions are scored from 1 ("strongly disagree") to 5 ("strongly agree"). High total scores for identity, timeline, consequences and emotional representations represent strong beliefs regarding number of symptoms attributed to the disorder, the chronic/cyclical nature of the disorder, adverse consequences and negative emotional association, respectively. High control and coherence scores indicate positive perceived controllability and good levels of understanding. Cronbach's $\alpha$ was 0.75 for identity and 0.79 for all other subscales. This questionnaire was only relevant for cough patients.

\section{Procedure}

After consenting to take part, participants could complete the questionnaire pack at hospital or at home (and return by post). Participants were also asked whether they would be happy to pass an adapted pack on to a non-cough friend. For all questionnaires, participants responded by choosing the corresponding scores or statements for each item.

The study received ethical approval from the NRES Committee North East - Tyne and Wear South.

\section{Data handling and analysis}

Missing data on an individual item level were handled by mean substitution within a subscale, in accordance with advice given by each measure. On a subscale/questionnaire level, multiple imputation $(\mathrm{n}=20)$ was undertaken, which acted as a sensitivity analysis. 
Multivariate analysis of variance (MANOVA) statistical analyses were conducted on the following dependent variable groupings: 1) personality, 2) anxiety, depression, fatigue and somatic symptoms, and 3) illness representations. Univariate outputs were checked and/or multiple imputation data were consulted in the case of a non-significant MANOVA result. This was to ensure that a non-significant difference between groups at a multivariate level of analysis did indeed relate to non-significant differences between groups on individual dependent variables, rather than being due to the lower sample size and subsequent reduction in power, and therefore sensitivity, to detect an effect between groups at the multivariate level. Where appropriate, analysis of variance (ANOVA) tests, t-tests and Tukey post hoc analyses were conducted to ascertain differences between groups for individual dependent variables. To avoid type 1 error inflation, $\alpha$ levels were adjusted using Bonferroni adjustment. If results were non-significant at the adjusted $\alpha$ level but were below the unadjusted level, they were still considered for further analyses, and tentative conclusions were drawn.

Data checks were conducted on the original data set. Multivariate normality was judged to be adequate through the use of standardised residual plots. Inter-variable correlations indicated there was no multi-collinearity. Homogeneity of variance (univariate) was assessed by comparing group variances, and, on the whole, these did not differ across the three groups. Statistics are reported accordingly, stated as equal variances assumed or not assumed. Box's $M$ test assessed the multivariate homogeneity of covariance (this test is highly sensitive so significance values would need to be highly significant to suggest heterogeneity, which they were not). Leverage values were plotted to assess multivariate outliers and illustrated that none were present.

\section{Results}

\section{Participants}

In total, 100 chronic cough and 72 non-cough packs were distributed. Response rates were $70 \%(n=67)$ and $30.5 \%(n=22)$, respectively. The overall sample of 89 participants consisted of 25 CRC patients, 42 explained cough patients and 22 non-cough participants (see table 1 for sample demographics). Explained cough diagnoses included asthma (38\%), gastro-oesophageal reflux disease (14\%), bronchiectasis, rhinitis, angiotensin-converting enzyme inhibitor use, Sjögren's associated interstitial lung disease, rheumatoid arthritis, eosinphilic bronchitis, COPD, post-infectious cough, sleep apnoea, or a combination of these. Details of current medications were not collected.

\section{Personality}

Using multivariate analyses, there was no statistically significant difference in the personality variables based on participants' cough grouping, $F(10,156)=1.363, \mathrm{p}=0.202$; Wilks' $\lambda=0.846$, partial $\eta^{2}=0.080$. However, univariate analyses indicated there may be a difference between groups on the Neuroticism dimension, $F(2,82)=3.341, \mathrm{p}=0.04$; partial $\eta^{2}=0.075$ (adjusted $\alpha$-level 0.01). Post hoc analyses showed that refractory coughers reported higher Neuroticism scores than non-coughers $(p=0.038)$, but this did not reach significance at the adjusted $\alpha$-level.

\section{Anxiety, depression, fatigue and somatic symptoms}

Multivariate analyses on the combination variable consisting of anxiety, depression, fatigue and somatic symptoms indicated there was a statistically significant difference between cough groups; $F(8,156)=3.384$, $\mathrm{p}=0.001$; Wilks' $\lambda=0.726$, partial $\eta^{2}=0.148$. Subsequent MANOVAs were conducted to analyse paired group contrasts. There was a significant difference between refractory coughers and explained coughers, $F(4,59)=3.170, \mathrm{p}=0.02$; Wilks' $\lambda=0.823$, partial $\eta^{2}=0.177$. There was a significant difference between refractory coughers and non-coughers, $F(4,40)=5.569, \mathrm{p}=0.001$; Wilks' $\lambda=0.642$, partial $\eta^{2}=0.358$. There was no significant difference between explained coughers and the non-cough group, $F(4,54)=1.656$, $\mathrm{p}=0.174$; Wilks' $\lambda=0.891$, partial $\eta^{2}=0.109$; however, univariate analyses were still consulted to ensure a potentially significant result was not missed.

\begin{tabular}{lccc|}
\hline TABLE 1 Participant demographics & & \\
& & Group & \\
\cline { 2 - 4 } & Refractory cough & Explained cough & Non-cough \\
\hline $\begin{array}{l}\text { Female } \mathbf{n} \\
\text { Male } \mathbf{n}\end{array}$ & 19 & 24 & 18 \\
Age years mean \pm sD & 6 & 18 & 4 \\
\end{tabular}


Univariate analyses were used to investigate the group differences for each of the four variables (adjusted $\alpha=0.0125$ ). These are presented in the following sections (see table 2 for mean and standard deviation values).

Anxiety

Refractory coughers were more anxious than non-cough participants, $F(1,43)=6.455, \mathrm{p}=0.015$, partial $\eta^{2}=0.131$ (approaching significance at the adjusted $\alpha$-level).

$52 \%$ of refractory coughers displayed a clinical level of anxiety, the majority falling within the "moderate" category (table 3). In comparison, $38 \%$ of explained coughers and $23 \%$ of non-coughers scored above the cut-off.

\section{Depression}

Refractory coughers were significantly more depressed than explained coughers, $F(1,62)=10.287, \mathrm{p}=0.002$, partial $\eta^{2}=0.142$, and non-cough participants, $F(1,43)=15.321, \mathrm{p}<0.001$, partial $\eta^{2}=0.263$.

$44 \%$ of refractory coughers recorded depression scores above the clinical cut-off, with $36 \%$ of the group displaying mild levels of depression. In comparison, $14 \%$ and $9 \%$ of explained and non-coughers, respectively, displayed mild levels of depression. None was moderately or severely depressed (table 3).

\section{Fatigue}

Refractory coughers were significantly more fatigued than explained coughers, $F(1,62)=10.351, \mathrm{p}=0.002$, partial $\eta^{2}=0.143$, as well as non-cough participants, $F(1,43)=21.248, \mathrm{p}<0.001$, partial $\eta^{2}=0.331$ (figure 1). Further consultation of the univariate result after multivariate analyses illustrated that explained coughers were more fatigued than non-cough participants, $F(1,57)=4.655, \mathrm{p}=0.035$, partial $\eta^{2}=0.075$, but this did not reach significance at the adjusted $\alpha$-level.

\section{Somatic symptoms}

Refractory coughers reported significantly more somatic symptoms than non-cough participants, $F(1,43)=$ 13.663, $\mathrm{p}=0.001$, partial $\eta^{2}=0.241$. Explained coughers also reported more somatic symptoms than non-cough participants, $F=(1,57), \mathrm{p}=0.02$, partial $\eta^{2}=0.091$, but this did not reach significance at the adjusted $\alpha$-level.

\section{Illness representations}

Multivariate analyses indicated no significant difference between refractory and explained coughers regarding their illness perceptions, $F(8,38)=1.357, \mathrm{p}=0.247$; Wilks' $\lambda=0.778$, partial $\eta^{2}=0.222$. However, the majority of analyses after multiple imputation illustrated a significant difference $(n=14)$, so univariate

\section{TABLE 2 Descriptive statistics by group}

\begin{tabular}{|c|c|c|c|c|}
\hline \multirow[t]{2}{*}{ Questionnaire } & \multirow[t]{2}{*}{ Variable } & \multicolumn{3}{|c|}{ Group } \\
\hline & & $\begin{array}{c}\text { Chronic } \\
\text { refractory cough }\end{array}$ & Explained cough & Non-cough \\
\hline \multirow{3}{*}{ Big Five Inventory } & Agreeableness & $4.06 \pm 0.59$ & $4.25 \pm 0.51$ & $4.14 \pm 0.64$ \\
\hline & Conscientiousness & $3.88 \pm 0.74$ & $4.16 \pm 0.60$ & $4.25 \pm 0.66$ \\
\hline & Neuroticism & $3.11 \pm 1.06^{*}$ & $2.67 \pm 0.74$ & $2.47 \pm 0.85$ \\
\hline Hospital Anxiety and Depression Scale & Depression & $6.56 \pm 4.12^{* \#}$ & $3.88 \pm 2.78$ & $2.55 \pm 2.74$ \\
\hline Chalder Fatigue Scale & Fatigue & $19.96 \pm 6.69^{* \#}$ & $15.48 \pm 5.68^{+}$ & $12.50 \pm 5.24$ \\
\hline Patient Health Questionnaire-15 & Physical symptoms & $10.48 \pm 6.02 *$ & $8.15 \pm 5.90^{+}$ & $4.60 \pm 4.22$ \\
\hline \multirow[t]{4}{*}{ Illness Perception Questionnaire-Revised } & Identity & $4.20 \pm 2.78$ & $4.32 \pm 3.42$ & \\
\hline & Timeline (chronic) & $21.17 \pm 5.73$ & $18.66 \pm 5.96$ & \\
\hline & Consequences & $18.83 \pm 5.32^{\#}$ & $15.77 \pm 4.49$ & \\
\hline & Emotional representations & $18.42 \pm 5.27^{\#}$ & $15.36 \pm 4.09$ & \\
\hline
\end{tabular}

Data are presented as mean \pm SD. ${ }^{*}: p<0.05$ between $C R C$ and non-cough; ${ }^{*}: p<0.05$ between $C R C$ and explained cough; ${ }^{+}: p<0.05$ between explained cough and non-cough. 


\section{TABLE 3 Anxiety and depression cut-off score frequencies by group}

\section{Cut-off values}

Anxiety

\section{Refractory cough ${ }^{\#}$}

\section{Explained cough"}

$12(48 \%)$
$2(8 \%)$
$10(40 \%)$
$1(4 \%)$

$26(62 \%)$
$8(19 \%)$
$6(14 \%)$
$2(5 \%)$

Non-cough $^{+}$
$17(77 \%)$
$3(14 \%)$
$2(9 \%)$
0

Depression

Refractory cough

$14(56 \%)$
$9(36 \%)$
$1(4 \%)$
$1(4 \%)$

\section{Explained cough"}

$36(86 \%)$

$6(14 \%)$

0
Non-cough ${ }^{+}$

$20(91 \%)$

$2(9 \%)$

0

${ }^{\#}: \mathrm{n}=25 ;{ }^{\text {ๆ }}: \mathrm{n}=42 ;^{+}: \mathrm{n}=22$.

analyses were consulted. The individual significance values of illness coherence, consequences and emotional representations were significant or approaching significance in the original data set and were all significant in the majority of the multiple imputation data analyses.

Univariate paired contrasts were therefore conducted. These showed that refractory coughers held significantly more negative consequence beliefs than explained coughers, $\mathrm{t}(61)=2.451, \mathrm{p}=0.017$, partial $\eta^{2}=0.090$. They also associated significantly more negative emotions with their cough than explained coughers, $t(61)=2.580$, $\mathrm{p}=0.012$, partial $\eta^{2}=0.098$, and had significantly lower illness coherence in comparison to explained coughers, $\mathrm{t}(61)=-2.557, \mathrm{p}=0.013$, partial $\eta^{2}=0.097$ (Bonferroni adjusted $\alpha=0.0167$ ) (equal variances assumed).

\section{Discussion}

People with chronic cough in general reported higher levels of fatigue and somatic symptoms than non-coughers. Refractory coughers specifically differed significantly from non-cough participants, reporting higher levels of depression, anxiety, fatigue and somatic symptoms. In comparison to participants with explained cough, refractory coughers scored significantly higher for fatigue and depression. Significant differences between the two cough samples were also found for the illness representation dimensions: consequences, illness coherence and emotional representations. Personality dimensions did not, at the most rigorous level of analysis, differ between groups.

The mean anxiety score of refractory coughers $(M=8.40)$ was slightly higher than those reported by sufferers of conditions such as IBS ( $M=6.8$ [28] and $M=7.0$ [29]). Given that members of the explained cough group have received an underlying diagnosis, a significant difference in anxiety between the two cough groups might be expected. This is what MCGARveY et al. [5] reported when comparing state anxiety in patients classified as idiopathic or treated cough. Although mean levels were higher in the CRC group than in the explained cough group in the present study, this did not reach significance. The diagnoses of the explained coughers could provide some explanation for this, as elevated anxiety is well recognised in, for instance, COPD [30], asthma and bronchiectasis [31]. The high proportion of refractory coughers displaying moderate levels of anxiety however, is in line with the conclusion of McGarvey et al. [5] conclusion of increased levels of psychomorbidity within this patient population.

Depression did differ by cough group, and mean scores for refractory cough $(\mathrm{M}=6.56)$ were comparable or higher than those reported in IBS studies (7.1 [28] and 3.9 [29]). Mean scores did not, however, reach what are thought of as clinically significant levels $(\geqslant 8)[26]$. The study by DicPinigaitis et al. [6] found that

FIGURE 1 Graph illustrating the differences in fatigue across the three groups. CRC: chronic refractory cough; Ex: explained cough. ${ }^{* *}: p=0.01 ;{ }^{* *}: p<0.001$.

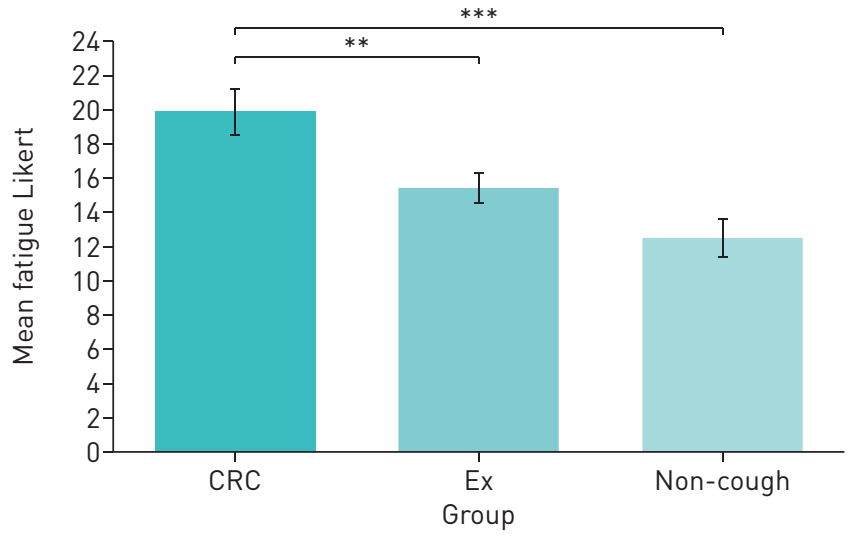


over $50 \%$ of patients reported significant depressive symptomatology. Methodology may explain these disparate findings. DicPinigaitis et al. [6] used the CES-D scale, which has one cut-off value and does not distinguish mood severity, and the reported mean is at the lower end of their depressive symptomology scale. As such, mood levels may be more similar than they appear and mild depression may be associated. This conclusion is supported by the fact that $44 \%$ of the refractory cough sample displayed levels of depression considered clinically significant, the majority scoring within the mild range.

Illness representations differed significantly between cough groups. Low illness coherence may highlight the difficulty of managing an "unexplained" condition. FrANK [32] has pointed out the importance to wellbeing of coherent narratives in chronic illness, and how chaos narratives (i.e. where individuals cannot make sense of their symptoms) are associated with more distress. As KIRMAYER et al. [33] suggested in their MUS studies, sense making is a transaction between the individual, publically available illness narratives and health professionals. As such, failed coherence is a systemic problem rather than an individual fault. It could be argued that increased anxiety and depression, perception of serious consequences and increased emotional response may result from this lack of coherence around cough, although of course in a cross-sectional study such causal links are speculative. We do know, however, that higher perceptions of negative illness beliefs have been associated with worsened health outcomes in other conditions, such as rheumatoid arthritis and psoriasis [34] and IBS [20, 21]).

Fatigue was significantly higher in CRC than in explained coughers. As a fatigue-specific measure has not been used in cough populations before, reference to other conditions provides a useful indicator of clinical significance. Levels of fatigue reported for CRC in this study $(M=19.96)$ are below fatigue-based conditions such as CFS $(M=28$ [35]). However, they are very similar to values reported in functional dysphonia $(\mathrm{M}=17$ [36]) and multiple sclerosis (20.94 [37]), the latter being a noteworthy comparison due to the extent that fatigue is recognised as a prominent symptom and treatment target. This is a novel finding in the CRC literature. The significant difference between the two cough groups warrants further investigation; it suggests heightened fatigue in CRC may not be accounted for purely by the presence of cough. The association between cough and sleep apnoea may be relevant here [38].

It must be noted, however, that rather than considering the involvement of such psychological factors with CRC in isolation, it is likely that they interact, both with each other and with the cough symptoms over time. Models of MUS have been proposed, such as the cognitive behavioural model of MUS [39], which describe how psychological factors, including those in this study, may be involved. For example, experiencing a cough but not being able to pinpoint the cause may result in reduced illness coherence and increased distress (particularly in vulnerable individuals). Patients' attention is then heightened to cough sensations, modulating the urge to cough and increasing further coughing episodes. Extended neural activation leads to sensitisation, reducing the cough threshold and further increasing the susceptibility of an individual to experiencing cough symptoms. Although this is a hypothetical vicious cycle, a similar framework with specific reference to cough has been described by VAN DEN BERGH et al. [40], providing a much needed platform for further research in this area.

A strength of the study was the inclusion of three groups, allowing comparison between refractory and explained coughers and a local "normal" non-cough group. Although relatively small, the sample sizes are comparable to other studies in this area $[5,6]$, and appropriate statistical adjustments were made to minimise possible power issues. Due to the cross-sectional study design, caution is advised when drawing conclusions. A prospective study would provide further insight into the stability of the associations and could identify predictor variables following a known trigger (i.e. respiratory infection). For example, a similar study identified anxiety, negative illness beliefs and other somatic symptoms at the time of infection as risk factors for IBS [29]. Similarly, a longitudinal study following coughers from first presentation, i.e. pre-diagnosis, through to successful treatment (in the case of explained coughers) or no resolution (in the case of unexplained coughers) would provide a more in-depth insight into the involvement and interaction of psychological factors in different illness trajectories. This would also allow for data collection at consistent and comparable points within the cough treatment period, for all cough patients. The non-cough comparison group also warrants consideration. Accessing a comparison group of similar age and socioeconomic status is difficult, so our group arose as a result of pragmatic sampling. We recognise this is a limitation, as we do not know much about this group. We stipulated that they should not have a chronic cough or were not attending hospital appointments, in an effort to create a "healthy" sample. However, physical or psychological morbidity is something we could not fully control for in this instance.

In conclusion, CRC patients do differ significantly from both non-coughers and explained coughers on a variety of psychological measures, identifying possible avenues for further research and intervention targets. Training health professionals in how to help patients make sense of their symptoms may make interventions more effective. This collaborative sense-making narrative work has shown promise in other 
persistent physical symptoms [41]. Low mood and fatigue management strategies are also warranted, given the elevated level of both in this sample. Given that CRC is currently often poorly managed, targeting factors such as those highlighted may help patients improve their management of the cough and reduce the adverse impact it can have on their lives.

\section{Acknowledgements}

We are grateful to Caroline Potts and staff in the R\&D Dept at Northumbria Healthcare NHSFT. Thanks go to David Clark-Carter (Staffordshire University) for his statistical guidance and expertise. Thanks to Surinder Birring and Alyn Morice for helpful comments on the manuscript.

\section{References}

1 Morice AH, McGarvey LP, Pavord I. Recommendations for the management of cough in adults. Thorax 2006; 61: $11-24$.

2 McGarvey LP. Does idiopathic cough exist? Lung 2008; 186: 1, 78-81.

3 Brignall K, Jayaraman B, Birring S. Quality of life and psychological aspects of cough. Lung 2008; 186: 1, 55-58.

4 French CL, Irwin RS, Curley FJ, et al. Impact of cough on quality of life. Arch Intern Med 1998; 158: 15, $1657-1661$.

5 McGarvey LPA, Carton C, Gamble LA, et al. Prevalence of psychomorbidity among patients with chronic cough. Cough 2006; 2: p4.

6 Dicpinigaitis PV, Tso R, Banauch G. Prevalence of depressive symptoms among patients with chronic cough Chest 2006; 130: 6, 1839-1843.

7 Janssens T, Silva M, Davenport PW, et al. Attentional modulation of reflex cough. Chest 2014; 146: 135-141.

8 Janssens T, Brepoels S, Dupont L, et al. The impact of harmfulness information on citric acid induced cough and urge-to-cough. Pulm Pharmacol Ther 2015; 31: 9-14.

9 Hegland KW, Bolser DC, Davenport PW. Volitional control of reflex cough. J Appl Phys 2012; 113: 39-46.

10 Ando A, Smallwood D, McMahon M, et al. Neural correlates of cough hypersensitivity in humans: evidence for central sensitisation and dysfunctional inhibitory control. Thorax 2016; 71: 4, 323-329.

11 Vertigan AE, Theodoros DG, Gibson PG, et al. Efficacy of speech pathology management for chronic cough: a randomised placebo controlled trial of treatment efficacy. Thorax 2006; 61: 12, 1065-1069.

12 Chamberlain SAF, Birring SS, Clarke L, et al. Efficacy of a physiotherapy, speech and language therapy intervention (PSALTI) on health related quality of life (HRQOL) for patients with refractory chronic cough: a randomised control trial. Thorax 2014; 69: Suppl. 2, A78.

13 Craig P, Dieppe P, Macintyre S, et al. Developing and evaluating complex interventions: the new Medical Research Council guidance. $\mathrm{Br}$ Med J 2008; 337: a1655.

14 Gale C, Hagenaars SP, Davies G, et al. Pleiotropy between neuroticism and physical and mental health: findings from 108038 men and women in UK Biobank. Translat Psychiatr 2016; 6: 4: e791.

15 De Gucht V, Fischler B, Heiser W. Personality and affect as determinants of medically unexplained symptoms in primary care: a follow-up study. J Psychosom Res 2004; 56: 3, 279-285.

16 Whitehead WE, Palsson O, Jones KR. Systematic review of the comorbidity of irritable bowel syndrome with other disorders: what are the causes and implications? Gastroenterology 2002; 122: 1140-1156.

17 Ford AC, Forman D, Moayyedi P, et al. Cough in the community: a cross sectional survey and the relationship to gastrointestinal symptoms. Thorax 2006; 61: 975-979.

18 Kroenke K, Wood DR, Mangelsdorff AD, et al. Chronic fatigue in primary care. Prevalence, patient characteristics, and outcome. JAMA 1988; 260: 929-934.

19 Petrie KJ, Weinman J. Patients' perceptions of their illness. The dynamo of volition in healthcare. Curr Dir Psychol Sci 2012; 21: 1, 60-65.

20 Rutter CL, Rutter DR. Illness representation, coping and outcome in irritable bowel syndrome (IBS). $\mathrm{Br} J$ Health Psychol 2002; 7: 4, 377-391.

21 Ferreira NB, Morris P, Eugenicos MP, et al. The role of illness perceptions and acceptance in outcomes of irritable bowel syndrome [Abstract]. Psychol Health 2010; 25: 1, 33.

22 John OP, Srivastava S. The Big-Five trait taxonomy: history, measurement, and theoretical perspectives. In: Pervin LA, John OP, eds. Handbook of Personality: Theory and Research. New York, Guilford Press, 1999; pp. 102-138.

23 Cella M, Chalder T. Measuring fatigue in clinical and community settings. J Psychosom Res 2010; 69: 1, 17-22.

24 Kroenke K, Spitzer RL, Williams JBW. The PHQ-15: validity of a new measure for evaluating the severity of somatic symptoms. Psychosom Med 2002; 64: 258-266.

25 Zigmond AS, Snaith RP. The hospital anxiety and depression scale. Acta Psychiatr Scand 1983; 67: 6, 361-370.

26 Bjelland I, Dahl AA, Haug TT, et al. The validity of the hospital anxiety and depression scale: an updated literature review. J Psychosom Res 2002; 52: 2, 69-77.

27 Moss-Morris R, Weinman J, Petrie KJ, et al. The revised illness perception questionnaire (IPQ-R). Psychol Health 2002; 17: 1-16.

28 Cho HS, Park JM, Lim CH, et al. Anxiety, depression and quality of life in patients with irritable bowel syndrome. Gut Liver 2011; 5: 1, 29-36.

29 Spence MJ, Moss-Morris R. The cognitive behavioural model of irritable bowel syndrome: a prospective investigation of patients with gastroenteritis. Gut 2007; 56: 8, 1066-1071.

30 Puhan MA, Frey M, Buchi S, et al. The minimal important difference of the hospital anxiety and depression scale in patients with chronic obstructive pulmonary disease. Health Qual Life Outcomes 2008; 6: 46.

31 Kunik ME, Roundy K, Veazey C, et al. Surprisingly high prevalence of anxiety and depression in chronic breathing disorder. Chest 2005; 127: 1205-1211.

32 Frank AW. The Wounded Storyteller: Body, Illness, and Ethics. Chicago, University of Chicago Press, 2013.

33 Kirmayer LJ, Groleau D, Looper KJ, et al. Explaining medically unexplained symptoms. Can J Psychiatry 2004; 49: 663-672. 
34 Scharloo M, Kaptein A. Measurement of illness perceptions in patients with chronic somatic illness: a review of the literature. In: Petrie KJ, Weinman J. eds. Perceptions of Health and Illness: Current Research and Applications. London, Harwood Academic Publishers, 1997; pp. 103-135.

35 White PD, Goldsmith KA, Johnson AL, et al. Comparison of adaptive pacing therapy, cognitive behaviour therapy, graded exercise therapy, and specialist medical care for chronic fatigue syndrome (PACE): a randomised trial. Lancet 2011; 377: 823-836.

36 O'Hara J, Miller T, Carding P, et al. Relationship between fatigue, perfectionism, and functional dysphonia. Otolaryngol Head Neck Surg 2011; 144: 921-926.

37 Van Kessel K, Moss-Morris R, Willoughby E, et al. A randomized controlled trial of cognitive behaviour therapy for multiple sclerosis fatigue. Psychosom Med 2008; 70: 205-213.

38 Chan K, Ing A, Birring SS. Cough in obstructive sleep apnoea. Pulm Pharmacol Ther 2015; 35: 129-131.

39 Deary V, Chalder T, Sharpe M. The cognitive behavioural model of medically unexplained symptoms: a theoretical and empirical review. Clin Psychol Rev 2007; 27: 781-797.

40 Van den Bergh O, Van Diest I, Dupont L, et al. On the psychology of cough. Lung 2012; 190: 55-61.

41 Burton C, Lucassen P, Aamland A, et al. Explaining symptoms after negative tests: towards a rational explanation. J R Soc Med 2015; 108: 84-88. 